\title{
Le clonage du gène de la myopathie myotubulaire définit une nouvelle famille de tyrosine phosphatases
}

La myopathie myotubulaire (MTM) est une maladie rare liée au $\mathrm{X}$, qui se traduit par une hypotonie néonatale très sévère, entraînant presque toujours un décès par défaillance respiratoire dans la première année [1]. La biopsie musculaire documente la présence de petites fibres avec noyau central entouré d'un halo dépourvu d'éléments contractiles, ressemblant aux myotubes fotaux. Cela suggère un blocage de la maturation des fibres musculaires, caractérisé par la migration des noyaux à la périphérie. En 1990 le gène $M T M$ avait été localisé par analyse de liaison dans la région Xq28. La rareté de la maladie, sa létalité précoce, et la grande proportion de cas sporadiques rendaient difficile une cartographie génétique plus précise et, en 1993, la région candidate était encore de 8 mégabases. Une judicieuse combinaison d'analyse clinique, cytogénétique et histopathologique permettait l'identification d'une anomalie chromosomique touchant la région Xq28 chez une jeune patiente avec forme modérée de myopathie myotubulaire (A. Nivelon, S. Gilgenkrantz, M. Fardeau et al.). Cela fut l'événement déterminant pour la stratégie de clonage positionnel. N. Dahl et $\mathrm{LJHu}$, dans notre laboratoire, mettaient en évidence une délétion de plusieurs mégabases emportant les gènes FMR1 et IDS (syndrome de Hunter) en Xq27.3q28, et montraient que le chromosome délété était préférentiellement actif chez la patiente, expliquant le phénotype (les femmes vectrices de la maladie n'ayant normalement pas d'expression clinique). La comparaison des données de liaison et de l'analyse de

tion présente chez un patient avec syndrome de Hunter mais sans myopathie myotubulaire, a abouti rapidement à limiter la région candidate à $600 \mathrm{~kb}$ [2]. Une cartographie physique et transcriptionnelle de cette région était entreprise en collaboration avec l'équipe d'A. Poustka. En parallèle, nous testions systématiquement les sondes de la région dans un groupe de plus de 40 patients réunis par le Myotubular Myopathy Consortium (animé par C. Wallgren-Pettersson). Deux d'entre eux montraient des délétions partiellement recouvrantes, et définissaient un nouvel intervalle candidat de seulement $280 \mathrm{~kb}$. Ces deux patients étaient les seuls à présenter de sévères anomalies du développement sexuel, témoignant de la présence dans cet intervalle d'un autre gène impliqué dans ce phénotype additionnel [3]. Des gènes candidats furent identifiés par sélection des $\mathrm{ADNc}$, amplification d'exons, et séquençage et prédiction informatique d'exons. L'un deux fut retrouvé muté chez plusieurs patients MTM (mutations frameshift ou faux sens) [4].

Le gène $M T M$, d'expression ubiquitaire, code pour une protéine d'environ 600-650 acides aminés (la position du codon de début de transcription n'est pas déterminée), que nous avons appelée myotubularine. Celle-ci ne ressemble à aucune protéine de fonction connue, à l'exception d'un segment de 12 acides aminés conforme au consensus du site actif des tyrosine phosphatases. L'on pouvait s'interroger sur la signification d'une similitude aussi restreinte. L'analyse des bases de données de séquences montrait, de façon inattendue pour un gène de maladie affectant la maturation musculaire, une excellente conservation du gène dans la levure, et dans le vers $C$. elegans, sous forme de deux ORF (open reading frame) issus du séquençage systématique du génome de ces organismes. En particulier, une identité de $44 \%$ sur 320 acides aminés était retrouvée entre les séquences humaines et de levure. Le segment «tyrosine phosphatase» est complètement conservé entre les trois organismes, et est la cible de deux mutations faux sens retrouvées chez deux patients. Cela illustre l'importance fonctionnelle de ce motif, et renforce l'hypothèse d'une activité tyrosine phosphatase. L'analyse des bases de données d'EST (expressed sequence tags, étiquettes d'ADNc) montrait en outre que le génome humain comportait au moins trois autres gènes très analogues (dénommés MTMR1, 2, 3). MTMR1 est situé à moins de $100 \mathrm{~kb}$ du gène $M T M$. Il s'agit donc d'une nouvelle famille de tyrosine phosphatases (putatives), dont il faudra définir les fonctions. L'analyse des gènes homologues chez la levure et chez $C$. elegans pourrait se révéler informative. La myotubularine est elle impliquée dans une voie de transduction du signal importante pour la maturation des fibres musculaires? Quels en sont les effecteurs? Les réponses à ces questions pourraient ouvrir un champ nouveau d'investigation pour une phase très mal connue du développement du muscle.

L'identification du gène va permettre d'améliorer considérablement le conseil génétique. Dans les nombreux cas sporadiques, il était pratiquement impossible de déterminer si la mère était vectrice, ou s'il 
s'agissait d'une nouvelle mutation. L'identification des mutations permettra de résoudre ce problème. A l'heure actuelle, nous avons identifié plus d'une quinzaine de mutations, correspondant à $30 \%$ des familles analysées (chaque famille étant porteuse d'une mutation différente, comme souvent pour les maladies létales liées au X) [4] et C. GuiraudChaumeil et J. Laporte, résultats non publiés). Les dix mutations faux sens détectées jusqu'ici touchent toutes des acides aminés conservés dans la levure. Toutefois, la structure du gène n'étant pas entièrement connue, la recherche des mutations est encore incomplète. On ne peut pour le moment éliminer l'hypothèse que le gène homologue adjacent (MTMR1) puisse être impliqué dans certaines familles.

En conclusion, ce résultat illustre une fois de plus l'importance de reconnaître et d'analyser les patients «exceptionnels » et l'intérêt, pour la génétique humaine, des programmes de séquençage d'organismes «modèles» pourtant bien éloignés de l'homme.

1. Wallgren-Pettersson C, Clarke A, Samson F, Fardeau M, Dubowitz V, Moser H, Grimm T, Barohn RJ, Barth PG. The myotubular myopathies: differential diagnosis of the $\mathrm{X}$ linked recessive, autosomal dominant, and autosomal recessive forms and present state of DNA studies. J Med Genet 1995; 32: 673-9.

2. Dahl N, Hu W, Chery M, Fardeau M, Gilgenkrantz S, Nivelon-Chevalier A, Sidaner-Noisette I, Mugneret F, Gouyon JB, Gal A, Kioschis P, d'Urso $\mathrm{M}$, Mandel JL. Myotubular myopathy in a girl with a deletion at Xq27-28 and unbalanced X inactivation assigns the MTM1 gene to a $600 \mathrm{~kb}$ region. Am J Hum Genet 1995; 56: 1108-15.

3. Hu W, Laporte J, Kress W, Kioschis P, Siebenhaar R, Poustka A, Fardeau M, Metzenberg A, Janssen EA, Thomas N, Mandel JL, Dahl N. Deletions in Xq28 in two boys with myotubular myopathy and abnormal genital development define a new contiguous gene syndrome in a $430 \mathrm{~kb}$ region. Hum Mol Genet 1996; 5 : 139-43.
4. Laporte J, Hu LJ, Kretz C, Mandel JL, Kioschis P, Coy JF, Klauck SM, Poustka A, Dahl $\mathrm{N}$. A gene mutated in X-linked myotubular myopathy defines a new putative tyrosine phosphatase family conserved in yeast. Nature Genet 1996; sous presse.

\section{Jocelyn Laporte Jean-Louis Mandel}

Institut de Génétique et de Biologie Moléculaire et Cellulaire, Cnrs/ Inserm/ULP, CU de Strasbourg, BP 163, 67404 Illkirch Cedex, France.

\section{Remerciements}

Le travail effectué à l'IGBMC (Inserm/ Cnrs/Université Louis-Pasteur), a également bénéficié du soutien de l'AFM et du GREG. Nous remercions les membres du Myotubular Myopathy Consortium (soutenu par le European Neuro-Muscular Center et l'AFM) d'avoir permis l'analyse de nombreux patients. 